\title{
Thermophoresis of Carboxylic Nanotubes in Gaseous Atmosphere
}

\author{
Aleksey Bubenchikov ${ }^{1,2}$, Aleksandr Potekaev ${ }^{3}$, Mikhail Bubenchikov ${ }^{1}$, \\ Vladimir Korobitsyn" ${ }^{1}$, Ivan Klykov ${ }^{1}$ \\ ${ }^{1}$ Tomsk State University, Tomsk, Russia \\ ${ }^{2}$ Buryat State University, Ulan-Ude, Russia \\ ${ }^{3}$ Siberian Physical-Technical Institute, Tomsk State University, Tomsk, Russia \\ Email: alexy121@mail.ru, kanc@spti.tsu.ru, michael121@mail.ru, kva635133@mail2000.ru
}

Received November 17, 2013; revised December 24, 2013; accepted January 9, 2014

Copyright (C) 2014 Aleksey Bubenchikov et al. This is an open access article distributed under the Creative Commons Attribution License, which permits unrestricted use, distribution, and reproduction in any medium, provided the original work is properly cited. In accordance of the Creative Commons Attribution License all Copyrights (C) 2014 are reserved for SCIRP and the owner of the intellectual property Aleksey Bubenchikov et al. All Copyright (C) 2014 are guarded by law and by SCIRP as a guardian.

\section{ABSTRACT}

The present paper deals with motion of carbon nanotubes in a temperature gradient field. A determined-static theory of nanosized particles' thermophores is developed. Analytical expressions for thermophoretic velocity and force of ultramicroheterogeneous particles in a gaseous atmosphere under near-normal conditions are provided. The calculations performed according to the suggested theory, as applied to closed carbon nanotubes, found the value of dimensionless velocity of thermophoresis. In accordance with the proposed hypothesis, Waldman's limit is achieved, which is expressed in constancy of thermophoretic velocity within the interval of the Knudsen parameter change from 10 to 100. In addition, it is found out that under conditions defined below, velocity of thermophoresis is independent of the length of a carboxylic nanotube. A good agreement with experiments is reached, which makes it possible to assume correspondence of the theory to the physical truth.

\section{KEYWORDS}

Thermophoresis; Carboxylic Tube; Dynamics of Nanoparticles; Ideal Gas; Statistical Distribution; Monokinetic Model; Mathematical Modelling

\section{Introduction}

Thermophoresis of fine, yet not nanosized particles, has been reported in a large number of studies among which it is possible to point out the following scientific works of general character [1-5].

In this work, we follow the approach described in [6,7], which is used to determine the velocity of thermophoretic displacement of a particle in a temperature-gradient field, as well as the thermophoretic force acting on the moving sample particle, which is balanced by the force of particle drug under equilibrium conditions.

\section{Particle Thermophoresis Velocity}

Similar to the case of finding the force of particle drag [6], to calculate thermophoresis velocity it is enough to consider the process of momentum exchange between a particle and molecules being present in a layer of thick- ness $\lambda$ (in the layer surrounding the particle equal to the free path length of a molecule). Let a temperature change occur in one of the predetermined directions. Within the length equal to $\lambda$ these changes are insignificant but they entirely govern the processes of thermophoretic motion of particles. Let us populate the $\lambda$-layer with gas environment molecules, making sure their statistical distribution is homogeneous.

Therefore we state a stepped temperature change in our theory, the size of a step in this gradation also being equal to $\lambda$.

For example, Figure 1 shows eight temperature layers, the tube being placed within six of them. The two covering layers also contain some molecules included in the whole amount of those hitting the carboxylic tube. The number of temperature layers is not important in the suggested calculation technology. It may be equal to two, four, six, etc. 


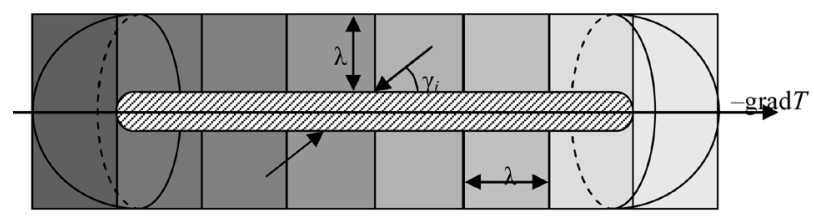

Figure 1 . The $\lambda$-layer surrounding a closed nanotube put into six temperature $\lambda$-layers.

Let us orient the tube along the temperature gradient and define the velocity of a particle moving under the action of thermophoretic force. Kinetic energy of translatory motion is distributed to three degrees of freedom, therefore

$$
\frac{3}{2} k T=\frac{m v^{2}(T)}{2} \text {. }
$$

From this equation, we get

$$
v(T)=\sqrt{\frac{3 k T}{m}} .
$$

Let vector grad $T$ be directed along the Oz-axis. Take a derivative with respect to the $z$-coordinates for both parts of Equation (2):

$$
\frac{\mathrm{d} v}{\mathrm{~d} z}=\frac{\mathrm{d} v}{\mathrm{~d} T} \frac{\mathrm{d} T}{\mathrm{~d} z}=\frac{1}{2} \sqrt{\frac{3 k}{m T}} \frac{\mathrm{d} T}{\mathrm{~d} z} .
$$

It is reasonable to assume that in the neighbourhood of an ultrafine or nanosized particle the behaviour of temperature variation is linear

$$
-\frac{\mathrm{d} T}{\mathrm{~d} z}=q=\text { const . }
$$

Replace the left-hand part of Equation (3) with finite differences that correspond to the velocity variation during a transition from one isothermal layer to another. Thus instead of Equation (3) we can approximately write

$$
\Delta v=\frac{\lambda}{2} \sqrt{\frac{3 k}{m T}} q .
$$

When determining the action of molecules on the particle let us use a scheme of equalized actions [6]. For this purpose let us divide the entire collection of molecules found in the $\lambda$-layer into counter-moving pairs. This will allow us to significantly simplify the calculation of the momentum exchange between the particle and the molecules. However, as it will be shown later, the statistically average result will be preserved. It should be noted that there is no particle velocity distribution in the Maxwellian representation in this model. In essence, it is a monokinetic (single-velocity) model.

Since within the path length shorter than $\lambda$ there is no molecular collision, it is reasonable to assume the temperature in every of the layers marked out and the velocities of thermal motion to be equal $v(T)$.

We have limited the amount of surrounding molecules by their amount in the $\lambda$-layer. However, it is not enough to perform the simplest calculation of thermophoresis velocity. Let us pick an elementary interaction act out of the whole collection of molecules interacting with the particle, in which a particle and a counter-moving pair of molecules participate. Therefore, we substitute all actual double collisions by model triple ones which do not cause Brownian motions. If we accept a regular pattern of particle reflection, the final result would represent a simple sum of interaction acts between the counter-moving pair and the particle.

\section{Balance of Momentum Projection on the Oz-Axis}

The top part of Figure 2 shows the projections of the counter-pair molecules' velocities onto the $\mathrm{Oz}$-axis prior to their collisions with the particle. The bottom part shows the velocity projections after a frontal elastic and regular reflection from the particle.

The balance of momentum projection onto the Oz-axis in a laboratory reference system for the case of a regular reflection is given by

$$
\begin{aligned}
& m v_{z}+m \Delta v_{z}-m v_{z}+M v_{P} \\
& =M v_{P}^{\prime}+m\left(v_{z}+v_{P}\right)-m\left(v_{z}+\Delta v_{z}-v_{P}\right) .
\end{aligned}
$$

Hence

$$
M \Delta v_{P i}=M\left(v_{P i}^{\prime}-v_{P i}\right)=2 m \Delta v_{z i}-2 m v_{P i} .
$$

Here $m, M$ are the masses of the molecule and the particle, respectively, and $v_{P i}^{\prime}, v_{P i}^{\prime}$ are the particle velocities prior to and after the collision with the countermoving pair

$$
\Delta v_{z i}=\Delta v \cos \gamma_{i} .
$$

Summing Equation (7) over all counter-moving pairs we obtain

$$
M\left(v_{P}^{\prime}-v_{P}\right)=n m \Delta v \sigma-n m v_{P} .
$$

Here $n$ is the number of molecules that have collided with the particle and $v_{P}$ is the average thermophoresis velocity of the tube prior to counter-moving pairs collisions in the $\lambda$-layer, $v_{P}^{\prime}=\frac{\sum_{i=1}^{n / 2} v_{P i}^{\prime}}{n / 2}$ is the average thermophoresis velocity of the tube after counter-moving

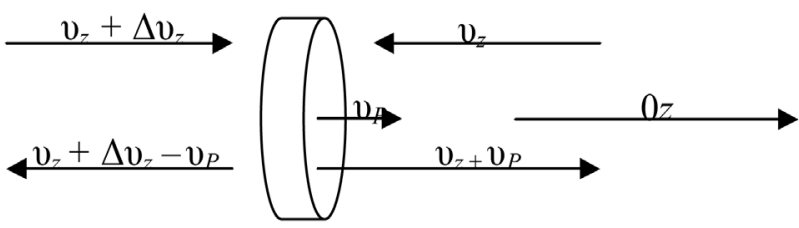

Figure 2. Elastic-contact interaction of a counter-moving pair with a moving particle. 
pairs collisions which is equal to $v_{P}$ under equilibrium conditions.

Taking into account that the left-hand part (Equation (9)) is equal to zero we get

$$
v_{P}=\sigma \Delta v \text {. }
$$

This velocity is the particle thermophoresis velocity. In Equation (10), $\sigma$ is found as

$$
\sigma=\frac{\sum_{i=1}^{n / 2} \cos \gamma_{i}}{n / 2}
$$

In cases of practical calculation we use the following formula for $\sigma$ :

$$
\sigma=\frac{\sum_{i=1}^{n}\left|\cos \gamma_{i}\right|}{n}
$$

Thus $\sigma$ is the average value of slope angle cosines modules of counter-moving pairs. Introducing $\Delta v$ from Equation (5) into Equation (10), we finally get

$$
v_{p}=\frac{\lambda \sigma}{2} \sqrt{\frac{3 k}{m T}} q
$$

\section{Calculation Statistics}

The $\lambda$-layer under consideration is found between the effective surface of the nanotube and the equidistant surface $\lambda$-spaced from it. Let us circumscribe a parallelepiped around the outer surface of the tube. Let us populate this parallelepiped with molecules of the gas surrounding the particle with the help of the random number generator used three times for every molecule, for the purpose of setting its spatial coordinates. Let us form a unit cube around every molecule and place a sampling particle into it in a random manner, i.e. use the random number generator three more times. Let us join this particle with the molecule, the resulting right line determining the direction of molecules motion in space. From here on we define $N$ as the number of molecules initially present in the $\lambda$-layer (Figure 1). For the time interval $\tau=\frac{\lambda}{v}$ we get $n$ which is the number of molecule-particle collisions. In this case, the fraction of molecules collided with the particle is defined by the equation $\delta=\frac{n}{N}$.

This value is included in the formulae used for defining particle resistance.

The value $\sigma$ determining thermophoresis is found as the average of the slope angle cosines modules of molecules trajectories against the temperature gradient direction. Calculating $\delta$ and $\sigma$ is the final stage of one test. The suggested number of such tests is 150, the values of $\delta$ and $\sigma$ being averaged thereafter.

\section{Orientation of Moving Particles}

Motion of nanotubes in gas is governed by the principle of least action or the principle of least constraint which are the same under the conditions of equilibrium. Both principles state the fact that in a gradient medium of molecules a nanotube is fixed along the direction grad $T$, i.e. it is subject to least resistance.

\section{Thermophoresis Velocity}

The particle motion velocity found in Equation (13) is actually the velocity of thermophoresis. As seen, it is independent of the number of molecular collisions (provided their number is sufficient to ensure proper statistical data for determination of $\sigma$ ) and is weakly dependant on the particle size. Within the Knudsen number range $K n=\frac{\lambda}{r_{p}} \in[10,100]$, which corresponds to proper nanoparticles, the thermophoresis velocity does not depend on the particle size and is solely determined by the number of atoms in a gas molecule and the values of temperature gradient and geometrical parameter $\sigma$ (see Equation (11)). Yu. V. Valtsyferov and S. M. Muradyan [2] state the following formula for the particle velocity due to thermophoresis:

$$
v_{p}=f \frac{v}{T} q,
$$

where $f$ is the dimensionless coefficient that depends on the Knudsen number and varies within the range of 0.05 1.56 and $v$ is the coefficient of kinematic gas viscosity.

Comparing Equation (13) and Equation (14), we obtain

$$
f=\frac{\lambda \sigma}{2 v} \sqrt{\frac{3 k T}{m}} .
$$

Statistical estimation for a spherical particle states $\sigma=$ 0.515. Introducing this value into the previous formula we obtain that $f=0.535$ which is in a good agreement with the measurement data and the theoretical results introduced in [11].

In order to take into account the occupied volume of the spherical particle in case $K n \geq 1$, we take into consideration four temperature layers, as shown in Figure 3.

In case $K n=1$, we get that $r_{p}=\lambda$, in cases when the Knudsen number has a greater value, the $r_{p}$-layer is considered in the same way as the $\lambda$-layer. Figure 4 shows the experimental data obtained for the dimensionless thermophoresis velocity of fine and ultrasized particles. The full curve line illustrates the calculation results.

Analogic complex distribution (the dashed line and the full curve line) can be obtained for the friction coefficient. In such a case, the dashed line will refer to Stockes distri- 


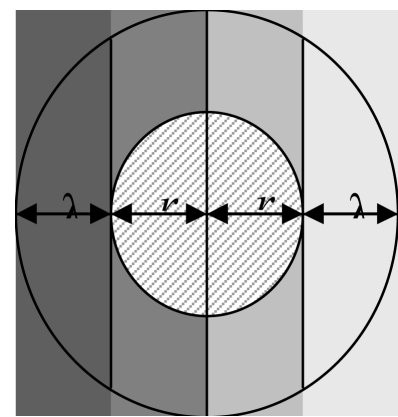

Figure 3. The occupied volume of the spherical particle taken into account.

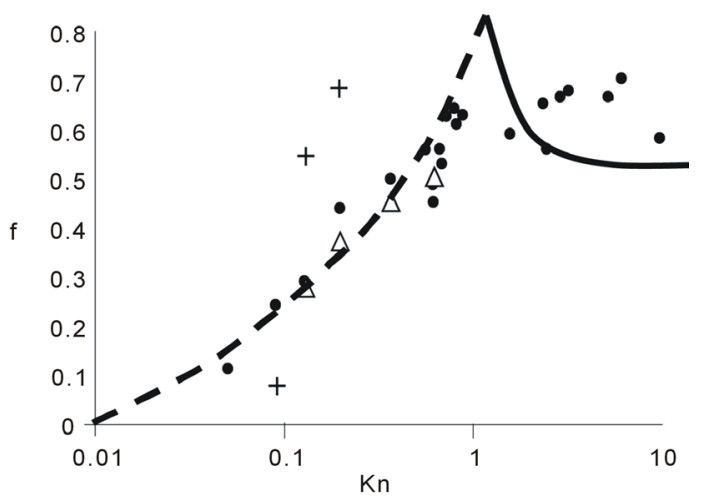

Figure 4. Dimensionless velocity of thermophoresis versus the Knudsen number. $\bullet-$ experimental results [8], $\Delta-\mathbf{e x}-$ perimental results [9], +- experimental results [10], - calculation results via proposed monokinetic theory, - - monotone approximation of experimental data. All data refer to spherical particles.

bution and the full curve line will refer to CunninghamMilliken-Davis data which agree with calculations via monokinetic theory [6].

\section{Thermophoretic Force}

Thermophoretic force can be defined on the basis of Newton's third law of motion, i.e. under the conditions of dynamic equilibrium

$$
F_{\text {т申 }}=\gamma v_{p},
$$

where $v_{p}$ is found from Equation (14) and the drag coefficient $\gamma$ was reported in [6,7]:

$$
\gamma=\frac{2}{3} \pi L \lambda v m N_{L} \frac{(K n+2)}{K n(K n+5)}, \quad K n \in[10,100]
$$

Here $v$ is the velocity of thermal molecular motion, $\lambda$ is the free lath length of a molecule, $K n$ is the Knudsen number, $N_{L}$ is the Loschmidt number under standard condition and $m$ is the molecular mass.

\section{Calculation Results}

The radius of the tube can be estimated in terms of the
Knudsen number: $K n=\frac{\lambda}{r_{P}}$. The lengths of the tubes included in calculations are given in Figure 5: $L=0.5 \lambda$ (the fine dashed line), $L=\lambda$ (the dashed line), $L=2 \lambda$ (the dot-and-dash line), $L=6 \lambda$ (the full line).

Thus in case $L>0.5 \lambda$, we discover that neither the lengths of carboxylic tubes nor their diameters influence $\sigma$, and consequently, the thermophoresis velocity. It is found that within the calculation range the changes of parameters are $\sigma=0.455$, which corresponds to the value of the dimensionless thermophoresis velocity of carboxylic tubes: $f=0.485$.

\section{Summary}

The present paper shows that the thermophoresis velocity
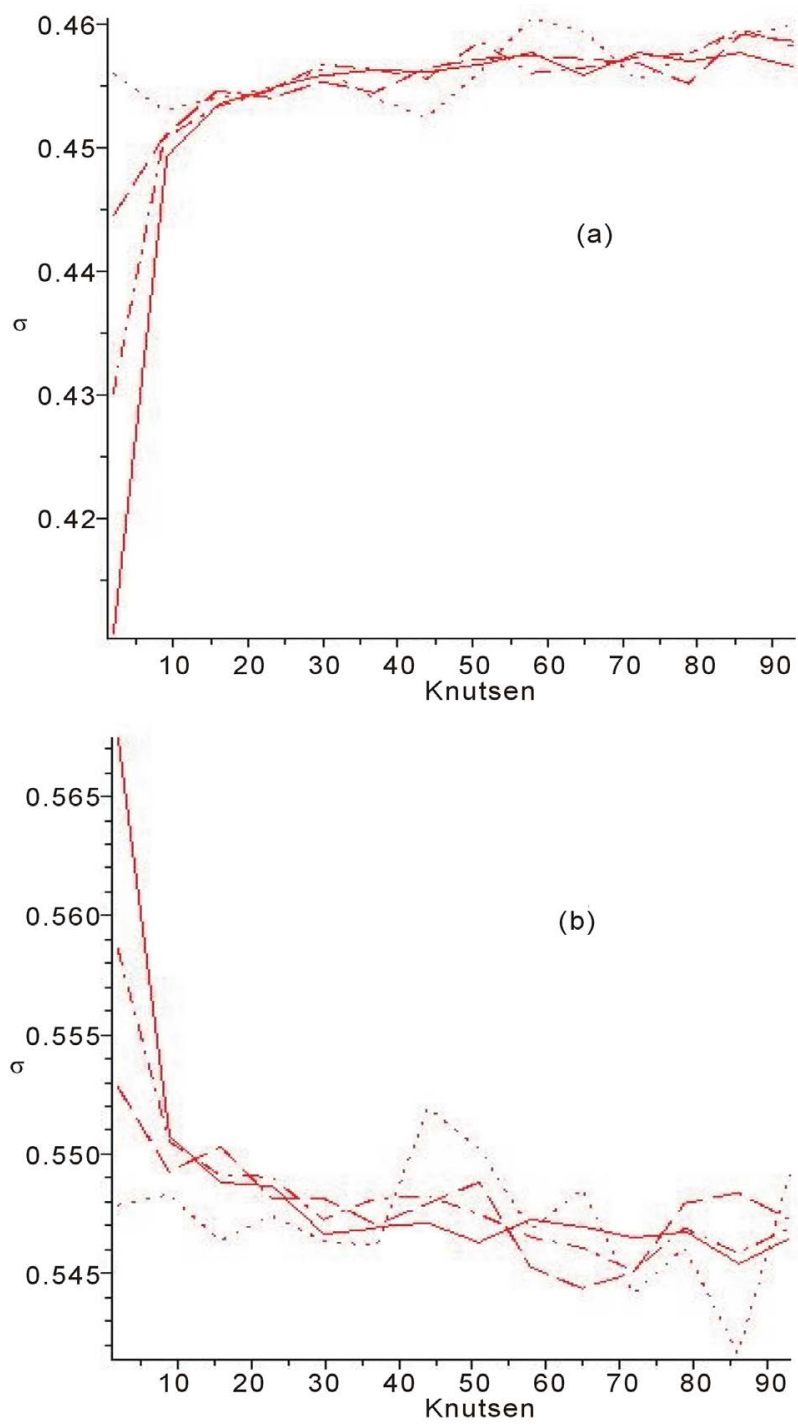

Figure 5. Distributions of geometrical parameter $\sigma$ for the tubes oriented along the temperature gradient field (a) and perpendicularly to it (b). 
of carboxylic tubes does not depend on the sizes of tubes $(10<K n<100)$ but is solely determined by the number of atoms in molecules of a gas medium and the temperature gradient in it, and the thermophoretic force acting on a particle being dependent on each of the above mentioned parameters. The calculations define that in cases of carboxylic tubes oriented along the temperature gradient field, the dimensionless coefficient in terms of linear relationship between the thermophoresis velocity and the temperature logarithm gradient is $f=0.485$.

\section{Acknowledgements}

The present scientific work was performed with financial support from Russian Fundamental Research Fund. Grant No 14-01-3165.

\section{REFERENCES}

[1] Z. R. Gorbis and F. E. Spokoinyi, “Физическая модель и математическое описание процесса движения мелких частиц в турбулентном потоке газовзвеси,” Teplofizika Vysokikh Temperatur, Vol. 15, No. 2, 1977, pp. 399-408.

[2] Y. V. Valsyferov and S. M. Muradyan, “Численный расчёт процессов тепломассопереноса при течении газа с частицами в прямолинейном цилиндрическом канале,” Teplofizika Vysokikh Temperatur, Vol. 22, No. 6, 1977, pp. 1152- 1157.

[3] S. P. Bakanov, “Термофорез вгазах при малых числах Кнудсена,” Uspekhi Fizicheskikh Nauk, Vol. 162, No. 9, 1992, pp. 133-152. http://dx.doi.org/10.3367/UFNr.0162.199209d.0133

[4] V. P. Redchits and Y. I. Yalamov, “Термофорез несфе- рической частицы в гидродинамическом режиме,” Physics Mathematics, Moscow Region State University, Moscow, No. 1, 2008, pp. 3-8.

[5] S. P. Bakanov, “О термофорезе в газах,,” Applied Mathematics and Mechanics, Vol. 69, No. 5, 2005, pp. 855-860.

[6] A. I. Potekaev, A. M. Bubenchikov and M. A. Bubenchikov, “Новые физические представления и метод описания и расчёта сопротивления движению малых частиц в газообазной среде,” Russian Physics Journal, Vol. 55, No. 12, 2012, pp. 54-61.

[7] M. A. Bubenchikov, A. I. Potekaev and A. M. Bubenchikov, “Три фундаментальные задачи молекулярной статистики,” Journal of Physics, Vol. 3, 2013, pp. 94100.

[8] E. A. Chernova, A. E. Turetskii, G. N. Lipatov and N. K. Kopyt, “Термофорез умеренно крупных частиц,” Physics of Airdispersed Systems: Interdepartmental Collected Works, Odessa, The I.I. Melchikov Odessa National University, Odessa, 2009, pp. 149-157.

[9] F. Prodi and G. Santacihara, "Measurements of Thermophoretic Velocities of Aerosol Particles in the Transition Region," Journal of Aerosol Science, Vol. 10, No. 4, 1979, pp. 421-425. http://dx.doi.org/10.1016/0021-8502(79)90037-5

[10] A. I. Storozhilova and G. I. Scherbina, "Измерение скорости термофореза крупных аэрозольных частиц и применение результатов измерения к определению коэффициентов теплового скольжения газа,” Doklady Akademii Nauk SSSR, Vol. 217, No. 2, 1974, pp. 386-389.

[11] L. Talbot, R. K. Cheng, R. W. Schefer and D. R. Willis, "Thermophoresis of Particles in a Heated Boundary Layer,” Journal of Fluid Mechanics, Vol. 12, No. 101, 1980, pp. 737-758. 